\title{
Prevention and Reversal of Cholera Enterotoxin Effects in Rabbit Jejunum by Nicotinic Acid
}

\author{
Nabila Turjman, Gerald S. Gotterer, and Thomas R. Hendrix, Departments of \\ Physiological Chemistry and Medicine, Johns Hopkins University School of \\ Medicine, Baltimore, Maryland 21205
}

A B S T RACT The cholera enterotoxin produces intestinal secretion associated with an elevation of tissue levels of cyclic adenosine $3^{\prime}, 5^{\prime}$-monophosphate levels of cyclic adenosine $3^{\prime}, 5^{\prime}$-monosphosphate (cAMP). The objectives of this study were to determine whether intestinal secretion and cAMP elevation induced by cholera toxin could be prevented, or once initiated, reversed by nicotinic acid, an agent known to lower tissue levels of cAMP. In rabbits, four jejunal loops were constructed as alternating control (3- $\mathrm{ml}$ isotonic electrolyte solution) and cholera toxin (same solution containing $50 \mu \mathrm{g}$ purified cholera toxin) loops. Net intestinal secretion was determined by measuring fluid accumulation, after which intestinal biopsies were taken for cAMP assay. The animals were pretreated either subcutaneously with $50 \mathrm{mg} / \mathrm{kg}$ nicotinic acid in saline $3 \mathrm{~h}$ and $1 \mathrm{~h}$ before the introduction of cholera toxin, or intraluminally with $200 \mathrm{mg} / \mathrm{kg}$ nicotinic acid in Ringer's lactate solution $15 \mathrm{~min}$ before the instillation of cholera toxin. Under these conditions, nicotinic acid blocked the cholera toxin-induced secretion and the rise in cAMP measured $3 \mathrm{~h}$ after the loops were exposed to cholera toxin. The effect of the nicotinic acid administered within the lumen on net intestinal secretion was studied. Maximal inhibition of net intestinal secretion was achieved with an intraluminally administered dose of nicotinic acid of $100 \mathrm{mg} / \mathrm{kg}$. This dose was chosen for testing the ability of nicotinic acid to reverse the effects of cholera toxin. When nicotinic acid was instilled into a fifth loop constructed distally to the four experimental loops $3 \mathrm{~h}$ after exposure of these loops to cholera toxin, both intestinal secretion and elevation of cAMP were reversed. These results suggest that nicotinic acid can prevent and reverse the secretory effects of cholera toxin and may have a role in

Presented, in part, at the Annual Meeting of American Society for Clinical Investigation, Washington, D. C., May 1977. Clin. Res. 25: 470A. (Abstr.)

Received for publication 8 August 1977 and in revised form 1 December 1977. the therapy of cholera and other cAMP-associated diarrheal diseases.

\section{INTRODUCTION}

It is generally held that cholera enterotoxin $(\mathrm{CT})^{1}$ produces intestinal secretion through the activation of intestinal adenylate cyclase with subsequent elevation of cyclic adenosine $3^{\prime}, 5^{\prime}$-monophosphate (cAMP) (1). If this view is correct, agents that inhibit adenylate cyclase or prevent elevation of levels of intestinal cAMP should prevent CT-induced secretion. In isolated fat cells studied in vitro, it has been shown that nicotinic acid is effective in preventing the rise in tissue cAMP elicited by a variety of stimuli such as epinephrine, ACTH, and glucagon (2).

The mechanism by which nicotinic acid prevents the rise in cAMP is controversial. Some hold that the effect is the result of the inhibition of adenylate cyclase (3), while others have presented evidence that the effect is the result of the activation of phosphodiesterase (4). Regardless of mechanism, it seemed worthwhile to determine whether CT-induced intestinal secretion and cAMP elevation could be prevented or, once initiated, reversed by nicotinic acid.

The results of the present study demonstrate that nicotinic acid, both by subcutaneous and intraluminal administration, is effective in preventing CT-induced secretion and elevation of tissue cAMP in in vivo jejunal loops of rabbits. In addition, these studies show that nicotinic acid can also reverse these intestinal effects of CT.

\section{METHODS}

Prevention of CT-induced changes. To determine the effect of pretreatment with nicotinic acid on CT-induced secretion, we compared net fluid movement and levels of cAMP in

${ }^{1}$ Abbreviations used in this paper: CAMP, cyclic adenosine 3',5'-monophosphate; CT, cholera enterotoxin; RL, Ringer's lactate. 
jejunal loops of rabbits pretreated with nicotinic acid and those receiving no drug. Male New Zealand white rabbits weighing between 1.7 to $2.3 \mathrm{~kg}$ were anesthetized with $20 \mathrm{mg} / \mathrm{kg}$ intravenous sodium pentobarbital after fasting overnight. The small intestine was exposed through a mid-line incision and a drain for duodenal juice was constructed. Four consecutive segments of jejunum measuring approximately $10 \mathrm{~cm}$ each in length were isolated with intact blood supply and cannulated. The loops were flushed clean with Ringer's lactate solution, emptied, and replaced in the abdominal cavity. Rectal temperature was monitored and the body temperature was maintained between $37^{\circ}$ and $39^{\circ} \mathrm{C}$ with the aid of a heat lamp. Throughout the study, hydration of the animals was maintained with an intravenous infusion of Ringer's lactate (RL) at the rate of $50-60 \mathrm{ml} / \mathrm{h}$. After a rest period of $1 \mathrm{~h}$ after the surgical procedures, a full-thickness intestinal biopsy was taken just distal to the fourth loop for base-line cAMP determination.

In each animal, the four intestinal loops were set up as alternating control loops exposed for $3 \mathrm{~h}$ to $3 \mathrm{ml}$ of isotonic electrolyte solution $\left(\mathrm{Na}^{+}, 150 \mathrm{mM} ; \mathrm{Cl}^{-}, 75 \mathrm{mM}\right.$; and $\mathrm{HCO}_{3}^{-}$, $75 \mathrm{mM}$ ), and CT loops similarly exposed to the same solution containing $50 \mu \mathrm{g}$ purified CT (lot BZ 2487, Schwarz Div., Mann Bector, Dickinson \& Co., Orangeburg, N. Y.). The order of the loops was varied, i.e., CT-control-CT-control or controlCT-control-CT. At the conclusion of all experiments, the volumes remaining in the loops were measured. Biopsies from each loop were taken for cAMP determination. The length of each loop was measured along the antimesenteric border after removal from the animal. Loops that were hemorrhagic were discarded from the study. The results were expressed as milliliters of net fluid movement per centimeter of loop length (net fluid movement $=$ final volume - initial volume [3 $\mathrm{ml}]$ ). Net secretion into the lumen was expressed as a positive value and net absorption from the lumen was expressed as a negative value. Mean values were obtained for control and CT loops in each animal by averaging the results of the duplicate loops in the same animal.

Subcutaneous injection. One group of nine rabbits (nicotinic acid rabbits) was injected subcutaneously with $50 \mathrm{mg} / \mathrm{kg}$ nicotinic acid in $8 \mathrm{ml} 0.9 \%$ saline $3 \mathrm{~h}$ and $1 \mathrm{~h}$ before the introduction of CT into the loops. This dose is an extrapolation of the dose used in man and rabbits in previously reported antilipolytic studies (5). In view of the relative insolubility of nicotinic acid in aqueous solution (6), nicotinic acid was dissolved in boiling water and maintained at approximately $40^{\circ} \mathrm{C}$ to prevent crystallization of the nicotinic acid before injection. In the other group of six rabbits (saline rabbits), 8 $\mathrm{ml}$ saline alone was administered at the same time intervals used with the nicotinic acid rabbits. In both groups, control and CT solutions were then instilled into their alternate loops and incubation continued for $3 \mathrm{~h}$.

Intraluminal incubation. To determine whether nicotinic acid is effective by the intraluminal route, $50 \mathrm{mg} / \mathrm{kg}$ nicotinic acid in $4 \mathrm{ml}$ saline was instilled directly into each of the four intestinal loops of one group of rabbits (nicotinic acid rabbits) $15 \mathrm{~min}$ before the introduction of CT. In the other group of rabbits (saline rabbits) $4 \mathrm{ml}$ saline alone was instilled in the loops. After the 15-min interval, the nicotinic acid or saline was flushed out and replaced with $3 \mathrm{ml}$ of electrolyte or CT solutions as in the previous experiments. After $3 \mathrm{~h}$, the volume of intestinal contents and loop lengths was measured and intestinal biopsies were taken for CAMP assay as before.

Dose response of nicotinic acid. To determine the optimal dose of nicotinic acid administered by the intraluminal route on CT-induced secretion, the same procedure as in "intraluminal incubation" was followed. The nicotinic acid concentration used ranged between 2.5 and $200 \mathrm{mg} / \mathrm{kg}$ in each animal.

\section{Reversal of CT-induced changes}

The experimental design was similar to the previous experiments and utilized four alternating control and CT loops. In contrast, however, the nicotinic acid was administered by way of an isolated loop rather than directly in the experimental loops. A fifth loop, approximately $20 \mathrm{~cm}$ in length, was constructed distally to the experimental loops for the instillation of either $100 \mathrm{mg}$ nicotinic acid/ $\mathrm{kg}$ in $6 \mathrm{ml} \mathrm{RL}$ or $\mathrm{RL}$ alone. $3 \mathrm{~h}$ after $\mathrm{CT}$ and electrolyte solutions had been instilled into the experimental loops, a time at which CT-induced secretion is maximal, nicotinic acid or RL control solution was introduced into the distal $20-\mathrm{cm}$ loop. $1 \mathrm{~h}$ later, the residual volumes in the experimental loops and their lengths were measured and biopsies were taken for cAMP assay.

\section{Assays}

Full-thickness biopsies for cAMP assay at indicated times were immediately frozen in liquid nitrogen. cAMP was extracted with cold 6\% TCA. cAMP levels were assayed by a modification of the binding protein method of Brown et al. (7). Results are expressed as picomoles per milligram protein. Tissue protein was determined by the method of Lowry et al. (8) using standards of bovine serum albumin.

\section{Statistics}

Statistical analyses were performed by Student's $t$ test at $P<0.05$ for paired and unpaired data (9). All results are expressed as the mean $\pm 1 \mathrm{SE}$.

\section{RESULTS}

\section{Prevention of CT-induced secretion and elevation of $c A M P$}

Subcutaneous injection. In the saline-injected animals, the net fluid accumulation after $3 \mathrm{~h}$ was less in control (containing no CT) than in CT-exposed loops (control, $0.014 \pm 0.10$ vs. CT, $0.29 \pm 0.09 \mathrm{ml} / \mathrm{cm} ; P$ $<0.001$ ) (Fig. 1). CT also caused a rise in (AMP to levels of $15.15 \pm 2.22 \mathrm{pmol} / \mathrm{mg}$ protein in contrast to zero time levels of $8.18 \pm 1.02 \mathrm{pmol} / \mathrm{mg}$ protein and control loop levels of $8.56 \pm 2.49 \mathrm{pmol} / \mathrm{mg}$ protein $(P$ $<0.001)$. On the other hand, nicotinic acid pretreatment produced a striking decrease in the CT-induced secretion (control, $0.063 \pm 0.098$ vs. CT, $0.103 \pm 0.072$ $\mathrm{ml} / \mathrm{cm}$; NS). Nicotinic acid pretreatment also prevented CT-induced elevation of cAMP (control, $8.17 \pm 2.95$ vs. CT, $8.33 \pm 2.19 \mathrm{pmol} / \mathrm{mg}$ protein; NS). No statistical difference was found between control and CT loops in the nicotinic acid animals; in fact, there was no statistical difference between the CT loops in the nicotinic acid group and control loops in the saline animals.

Intraluminal instillation of nicotinic acid. To determine whether nicotinic acid pretreatment by the intraluminal route would prevent CT-induced intes- 

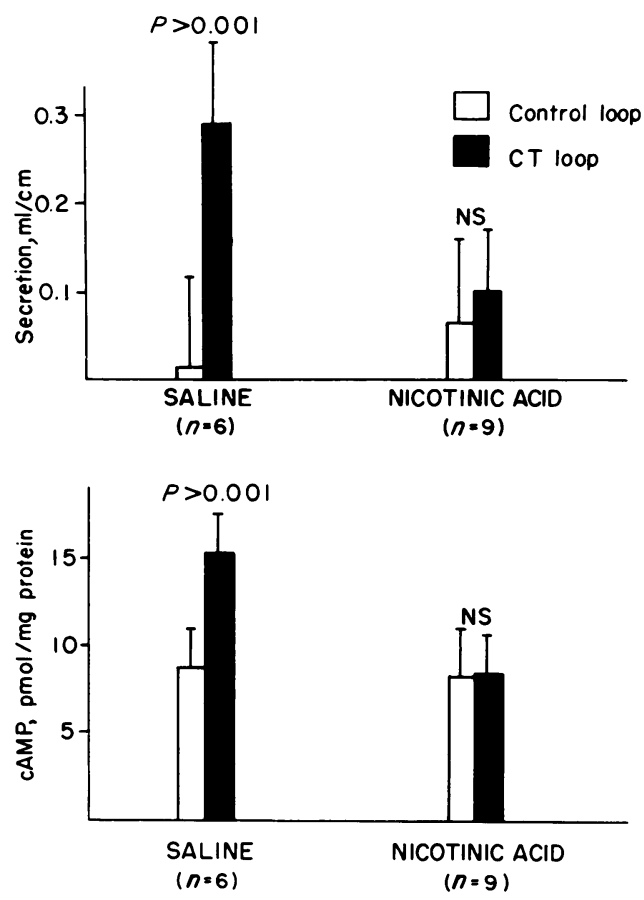

Figure 1 Effect of nicotinic acid pretreatment, $100 \mathrm{mg} / \mathrm{kg}$, administered subcutaneously on intestinal secretion (upper panel) and cAMP levels (lower panel) of in vivo rabbit jejunum. All measurements were made $3 \mathrm{~h}$ after $50 \mu \mathrm{g}$ of CT was inoculated into the experimental loops. CT induces secretion with concomitant rise in cAMP in animals injected with saline. In nicotinic acid-treated animals, there is no statistical difference between control and CT loops. Values are mean \pm SE. $P$ values above bars represent comparisons of control and CT loops in individual animals. $n$ represents the number of animals studied.

tinal secretion, $50 \mathrm{mg} / \mathrm{kg}$ nicotinic acid in $4 \mathrm{ml}$ saline was instilled directly in each intestinal loop for $15 \mathrm{~min}$ before the instillation of CT or control electrolyte solution. The results of these experiments are similar in every respect to those obtained by pretreatment with a subcutaneous injection. Both CT-induced intestinal secretion and rise in tissue cAMP were prevented by nicotinic acid. In these nicotinic acidpretreated animals, the rate of secretion and cAMP levels were not significantly different in control and CT loops (secretion-control, $0.18 \pm 0.070$ vs. CT; 0.23 $\pm 0.075 \mathrm{ml} / \mathrm{cm}$, NS; cAMP levels-control, $8.3 \pm 0.8$ vs. CT, $9.5 \pm 0.9 \mathrm{pmol} / \mathrm{mg}$, NS) (Fig. 2).

Effect of nicotinic acid dose by intraluminal route. The effect of nicotinic acid dose administered intraluminally on the inhibition of CT-induced secretion is shown in Fig. 3. Maximal inhibition was achieved with the dose of $100 \mathrm{mg} / \mathrm{kg}$. This dose was chosen for testing the ability of nicotinic acid to reverse the effects of CT.
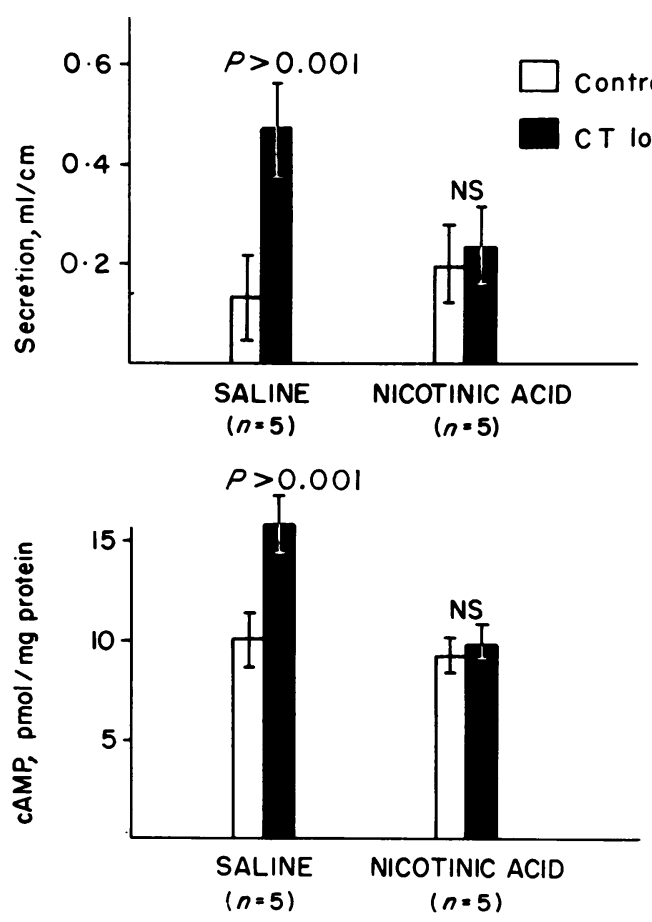

Figure 2 Effect of nicotinic acid pretreatment, $200 \mathrm{mg} / \mathrm{kg}$, administered by the intraluminal route on intestinal secretion and cAMP levels $3 \mathrm{~h}$ after CT introduction into rabbit jejunal loops. CT induces secretion $(P>0.001)$ and causes a rise in CAMP $(P>0.001)$ in animals treated with saline alone. In nicotinic acid-treated animals, there is no significant differences between control and CT loops in both intestinal secretion and cAMP levels. Values are mean \pm SE. $P$ values above bars represent comparison of control and CT loops in individual animals. $n$ represents the number of animals studied.

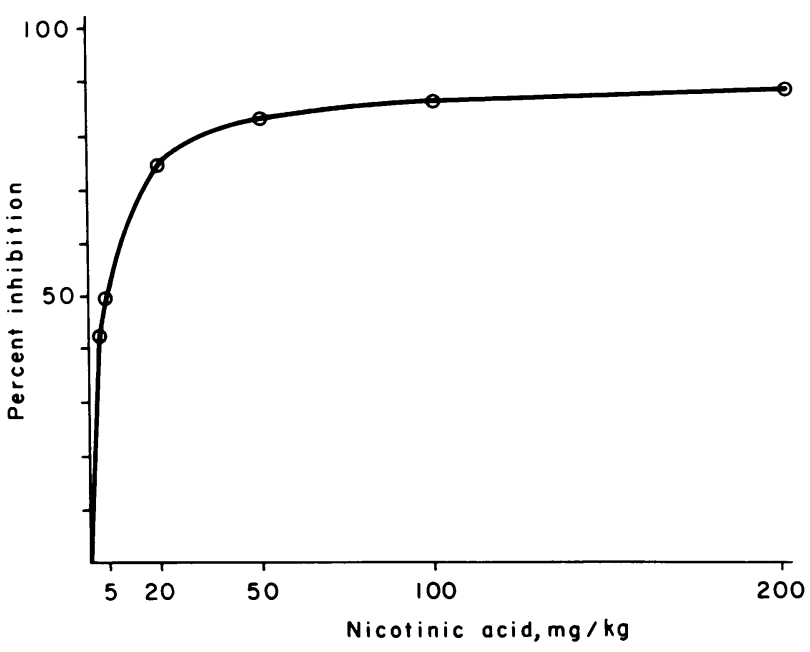

FIGURE 3 Dose-response relation of nicotinic acid on the inhibition of CT-induced intestinal secretion. Nicotinic acid was introduced into the experimental loops $15 \mathrm{~min}$ before the introduction of CT. Significant inhibition was detected at each dose of nicotinic acid with $50 \%$ inhibition occurring at a dose of $5 \mathrm{mg} / \mathrm{kg}$. 
Reversal of CT-induced secretion and elevation of cAMP by nicotinic acid

To determine whether nicotinic acid could reverse CT-induced intestinal secretion once it has been initiated, a significant net secretion was induced in $\mathrm{RL}$ animals. $4 \mathrm{~h}$ after exposure to $50 \mu \mathrm{g}$ of CT, a secretion of $0.37 \pm 0.09 \mathrm{ml} / \mathrm{cm}$ was obtained in CT loops in contrast to a secretion of $0.07 \pm 0.05 \mathrm{ml} / \mathrm{cm}$ in control loops $(P<0.001)$. Similarly, a concomitant rise in tissue levels of cAMP was obtained in CT loops $(17.16 \pm 1.16 \mathrm{pmol} / \mathrm{mg})$ whereas control loops were unchanged from zero time (control, $10.17 \pm 0.76$ vs. zero, $11.8 \pm 1.05 \mathrm{pmol} / \mathrm{mg}$; NS) (Fig. 4). On the other hand, after a single dose of $100 \mathrm{mg} / \mathrm{kg}$ nicotine acid was instilled into the distal $20-\mathrm{cm}$ loop $3 \mathrm{~h}$ after $50 \mu \mathrm{g}$ of CT had been instilled into the experimental loop, the 4-h fluid volumes and cAMP levels of CT loops were not significantly different from the values obtained in control loops which have not been exposed to CT (secretion-control, $0.08 \pm 0.06$ vs. CT, $0.09 \pm 0.04 \mathrm{ml} / \mathrm{cm}$, $\mathrm{NS}$; and cAMP-control, $11.80 \pm 1.19$ vs. CT, 11.88 $\pm 1.06 \mathrm{pmol} / \mathrm{mg}$, NS) (Fig. 4).

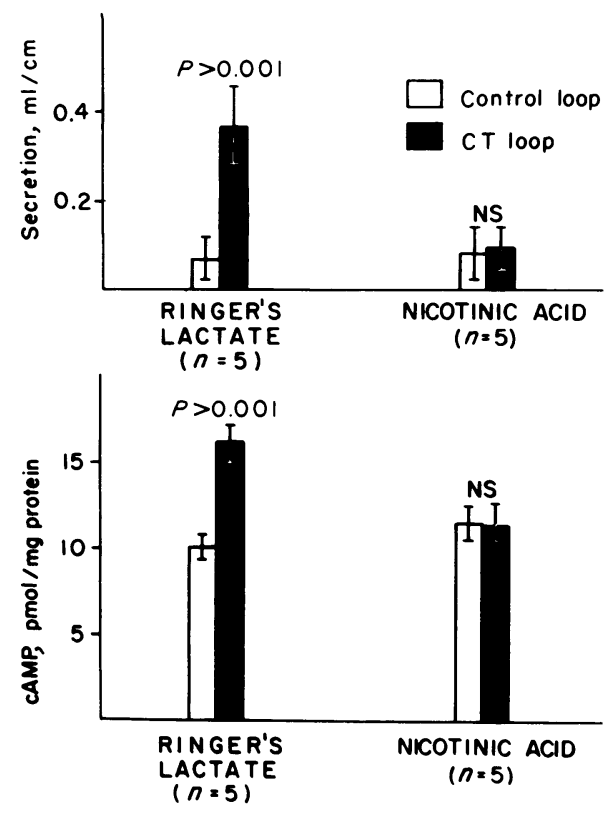

FIGURE 4 Effect of intraluminally administered nicotinic acid on established CT-induced secretion and cAMP levels. Nicotinic acid was introduced into a distal loop $3 \mathrm{~h}$ after CT was introduced into the experimental loop. All measurements were made $1 \mathrm{~h}$ after the administration of nicotinic acid. CT induces secretion $(P>0.001)$ and causes a rise in cAMP $(P>0.001)$ in animals treated with RL alone. In the nicotinic acid-treated animals, there is no significant difference between control and CT loops in either intestinal secretion or CAMP levels. Values are mean $\pm \mathrm{SE}$. $P$ values above bars represent comparisons of control and CT loops in individual animals. $n$ represents the number of animals studied.

\section{DISCUSSION}

Fecal water and electrolyte losses accompanying enterotoxigenic diarrhea can be replaced by the use of orally administered electrolyte solutions containing an actively transported solute such as glucose (10). Although therapy is simplified by minimizing the need for intravenous fluid replacement, the duration of diarrhea is not shortened by oral fluid replacement. If an agent capable of inhibiting enterotoxin-induced secretion could be identified that is effective orally, nontoxic, and inexpensive, the therapy of toxigenic diarrhea would be further simplified.

Although a number of agents have been found that will prevent CT-induced intestinal secretion such as cycloheximide (11), polymixin (12), indomethacin (13), aspirin (14), methyl prednisolone (15), ethacrynic acid (16), amphotericin B (17), and propranolol (18), only cycloheximide and methyl prednisolone have been shown to be able to reverse secretion. Our studies have demonstrated that nicotinic acid is also capable of reversing as well as preventing CT-induced secretion.

Butcher et al. (2) have shown that in isolated fat cells, nicotinic acid suppresses the elevated cAMP levels engendered by epinephrine, ACTH, and glucagon and thereby reverses the lipolytic effect of these agents. Nicotinic acid, but not nicatinamide, has been shown to be an effective anti-lipolytic agent (19). Our in vivo studies have shown that nicotinic acid is capable of preventing the elevation of intestinal cAMP and the associated secretion induced by CT. Extrapolating from studies on lipolysis, it is likely that this effect is the result of nicotinic acid rather than one of its metabolites.

Pretreatment of rabbits with nicotinic acid either parenterally or in the intestinal lumen prevents the expected intestinal secretion and elevation of intestinal cAMP that follows exposure to CT (Figs. 1 and 2). In addition the installation of nicotinic acid intraluminally reverses the effects produced by prior exposure to CT (Fig. 4). The small volume remaining at the 4th $\mathrm{h}$ in the CT loops of the nicotinic acidtreated animal ( $1 \mathrm{~h}$ after the introduction of nicotinic acid into the distal loop) was unexpected. It was anticipated that the 4 th-h volume in the CT loops of the nicotinic acid-treated animals would have been equivalent to the volume found in CT loops of control animals at $3 \mathrm{~h}$. Instead, reabsorption of secreted fluid was observed in the CT loops after instillation of nicotinic acid. These observations, therefore, indicate that not only was secretion inhibited but absorption was stimulated.

The present studies do not provide an explanation for the fluid absorption from the CT loop observed in the hour after the instillation of nicotinic acid. Since the final 
volumes in control loops in nicotinic acid-treated animals were similar to those in control loops in untreated animals, nicotinic acid stimulation of absorption cannot be advanced as an explanation of the findings. Although we have not observed nicotinic acid stimulation of absorption in control loops, we have observed an absorptive capacity equivalent to that observed in these experiments in random control animals. It is conceivable that the enhanced absorption seen in the CT loops of the nicotinic acid-treated animals is a result of the presence of a fluid with a more favorable composition than the electrolyte solution instilled into the control loops. Alternatively, CT may stimulate an absorptive process which under usual conditions of study is masked by the greater enhancement of the secretory process. Nicotinic acid may have disproportionately shut off the secretory process in contrast to its effect on the absorptive process. In support of this suggestion are data which show that CT increases absorptive flux (20). Furthermore, elevated levels of cAMP caused by theophylline or CT have been correlated with increased uptake of some neutral and dibasic amino acids (21). Although given little attention in the past, there is, therefore, evidence that CT may enhance absorption and this effect may provide an explanation for our unexpected observations.

The mechanism by which nicotinic acid exerts its effect on CT-induced secretion and elevation of cAMP is not clear. The question of mechanism has not been resolved in the more extensively studied in vitro adipocyte system. Skidmore et al. (3) have reported that nicotinic acid prevents hormonally induced elevation of cAMP by inhibiting adenylate cyclase, whereas Schwabe (4) attributes the effect to stimulation of phosphodiesterase. Since the steps between binding of CT to the intestinal epithelium, the subsequent elevation of CAMP and the stimulation of intestinal secretion have yet to be defined, it is not possible to pinpoint the site of action of nicotinic acid. Nevertheless, it is of interest that Gill and King (22) have presented evidence with pigeon erythrocytes that CT stimulates a NAD-requiring reaction that leads to the formation of a permanently activated adenylate cyclase. More recently, evidence has been presented invoking a NADase activity (23) for CT analogous to that which has been so elegantly proven for diphtheria toxin (24). Whether or not nicotinic acid or one of its metabolites inhibits the postulated CT NADase activity remains to be determined. Another mechanism by which CT increases adenylate cyclase activity has been reported to be prevention of the hydrolysis of the bond between adenylate cyclase and guanosine triphosphate, a step which has been proposed as the turn off mechanism that returns activated adenylate cyclase to the basal inactive state (25).

Regardless of which mechanism is acting, the re- sults of these experiments suggest that nicotinic acid might be effective in controlling diarrhea associated with cholera and possibly other diarrheal disorders associated with elevated levels of cAMP. Nicotinic acid has been used therapeutically in man as an antilipolytic agent. To what extent the effects of nicotinic acid described here, in rabbits, can be extrapolated to humans in the treatment of diarrheal diseases remains to be examined.

\section{ACKNOWLEDGMENTS}

We wish to thank Dr. Naji Sahyoun for his suggestions for potential inhibitors of adenylate cyclase.

This study was supported by Commonwealth Exchange Program W56-2002 and by National Institutes of Health grant AI-08187.

\section{REFERENCES}

1. Schafer, D. E., W. D. Lust, B. Sircar, and N. D. Goldberg. 1970. Elevated concentration of adenosine $3^{\prime}: 5^{\prime}-$ cyclic monophosphate in intestinal mucosa after treatment with cholera toxin. Proc. Natl. Acad. Sci. U. S. A. 67: 851-856.

2. Butcher, R. W., C. E. Baird, and E. W. Sutherland. 1968. Effects of lipolytic and antilipolytic substances on adenosine $3^{\prime}, 5^{\prime}$-monophosphate levels in isolated fat cells. J. Biol. Chem. 243: 1705-1712.

3. Skidmore, I. F., P. S. Schönhöfer, and D. Kritchevsky. 1971. Effects of nicotinic acid and some of its homologues on lipolysis, adenyl cyclase, phosphodiesterase and cyclic AMP accumulation in isolated fat cells. Pharmacology. (Basel). 6: 330-338.

4. Schwabe, U. 1971. Effect of nicotinic acid on $3^{\prime}, 5^{\prime}$ AMP-phosphate-diesterase activity in adipose tissue. In Metabolic Effects of Nicotinic Acid and its Derivatives. K. F. Gey and L. A. Carlson, editors. Hans Huber Publishers, Bern, Switzerland. 1st edition. 367-371.

5. The Pharmacological Basis of Therapeutics. 1970. L. S. Goodman and A. Gilman, editors. Macmillan, Inc., New York. 4th edition. 1654 .

6. The Merck Index. 1976. An Encyclopedia of Chemicals and Drugs. M. Windholz, editor. Merck \& Co., Inc., Rahway, N. J. 9th edition. 6343.

7. Brown, B. L., R. P. Ekins, and J. D. N. Albano. 1972. Saturation assay for cyclic AMP using endogenous binding protein. Adv. Cyclic Nucleotide Res. 2: 25-40.

8. Lowry, O. H., N. J. Rosebrough, A. L. Farr and R. J. Randall. 1951. Protein measurement with the Folin phenol reagent. J. Biol. Chem. 193: 265-275.

9. Snedecor, G. W., and W. G. Cochran. 1967. Statistical Methods. The Iowa State University Press, Ames, Iowa. 6 th edition. 593 pp.

10. Pierce, N. F., R. B. Sack, R. C. Mitra, J. G. Banwell, K. L. Baughman, D. S. Fedson, and A. Mondal. 1969. Replacement of water and electrolyte losses in cholera by an oral glucose-electrolyte solution. Ann. Intern. Med. 70: $1173-1176$.

11. Harper, D. T., Jr., J. H. Yardley, and T. R. Hendrix. 1970. Reversal of cholera extoxin-induced jejunal secretion by cycloheximide. Johns Hopkins Med.J. 1126: 258-266.

12. Maimon, H. N., W. E. Mitch, J. G. Banwell, and T. R. Hendrix. 1976. Inhibition of enterotoxin-induced intestinal secretion by the polypeptide antibiotic polymixin. Johns Hopkins Med. J. 138: 82-90. 
13. Wald, A., G. S. Gotterer, G. R. Rajendra, N. A. Turjman, and T. R. Hendrix. 1977. Effect of indomethacin on cholera-induced fluid movement, unidirectional sodium fluxes and intestinal cAMP. Gastroenterology. 72: 106110.

14. Finck, A. D., and R. L. Katz. 1972. Prevention of cholerainduced intestinal secretion in the cat by aspirin. Nature (Lond.). 238: 273-274.

15. Charney, A. N., and M. Donowitz. 1976. Prevention and reversal of cholera enterotoxin-induced intestinal secretion by methyl prednisolone induction of $\mathrm{Na}^{+}-\mathrm{K}^{+}$-ATPase . J. Clin. Invest. 57: 1590-1599.

16. Carpenter, C. C. J., G. T. Curlin, and W. B. Greenough. 1969. Response of canine Thirty-Vella jejunal loops to cholera exotoxin and its modification by ethacrynic acid. J. Infect. Dis. 120: 332-338.

17. Chen, L. C., R. L. Guerrant, J. E. Rohde, and A. G. T. Casper. 1973. Effect of amphotericin $B$ on sodium and water movement across normal and cholera toxinchallenged canine jejunum. Gastroenterology. 65: 252-258.

18. Taub, M., G. Bonorris, A. Chung, M. J. Coyne, and L. J. Schoenfield. 1977. Effect of propranolol on bile acidand cholera enterotoxin-stimulated cAMP and secretion in rabbit intestine. Gastroenterology. 72: 101-105.

19. Carlson, L. A. 1963. Studies on the effect of nicotinic acid on catecholamine-stimulated lipolysis in adipose tissue in vitro. Acta Med. Scand. 173: 719-722.

20. Grayer, D. T., H. A. Serebro, F. L. Iber, and T. R Hendrix. 1970. Effect of cycloheximide on unidirectional sodium fluxes exposure. Gastroenterology. 58: 815-819.

21. Kinzie, J. L., J. A. Ferrendelli, and D. H. Alpers. 1973. Adenosine cyclic $3^{\prime} 5^{\prime}$-monophosphate-mediated transport of neutral and dibasic amino acid in jejunal mucosa. J. Biol. Chem. 248: 7018-7024.

22. Gill, D. M., and C. A. King. 1975. The mechanism of action of cholera toxin in pigeon erythrocyte lysates. J. Biol. Chem. 250: 6424-6432.

23. Moss, J., V. C. Manganiello, and M. Vaughan. 1976. Hydrolysis of nicotinamide adenine dinucleotide by choleragen and its A protomer: Possible role in the activation of adenylate cyclase. Proc. Natl. Acad. Sci. U. S. A. 73: 4424-4427.

24. Kandel, J., R. J. Collier, and D. W. Chung. 1974. Interaction of fragment A from diphtheria toxin with nicotinamide adenine dinucleotide. J. Biol. Chem. 249: 20882097.

25. Cassel, D., and Z. Selinger. 1977. Mechanism of adenylate cyclase activation by cholera toxin: Inhibition of GTP hydrolysis at the regulatory site. Proc. Natl. Acad. Sci. U. S. A. 74: 3307-3311. 